\title{
Facilitadores e barreiras para a prática esportiva por parte de atletas com comprometimentos no Brasil \\ Facilitators and barriers for sports practice by athletes with impairments in Brazil \\ Facilitadores y barreras para la práctica deportiva de atletas con discapacidad en Brasil

\author{
Yasmin Vicente Vieira, Jackeline Colere, Doralice Lange de Souza \\ UniversidadeFederal do Paraná (Brasil)
}

\begin{abstract}
Resumo: 0 bjetivo do estudo foi o de explorar osfacilitadores easbarreirasparaapráticaesportivapor partede atletas com comprometimentos (ACC), dealto rendimento ebrasileiros, ativamenteenvolvidosem competiçõesdenível nacional el ou internacional. A pesquisafoi de caráter qualitativo e exploratório. A coletade dados foi feitaatravés de entrevistas semiestruturadas com noveACC de seis modalidades paralímpicas. Realizamos uma análise indutiva e temática dos dados. O s principais facilitadores citadosforam: apoio da família; exemplo de outrosACC através da mídia; socialização; ganhos financeiros com o esporte; incentivos por partede centros de reabilitação e de profissionais da saúde; possibilidade de conhecer novos lugares; gosto pelo esportee pela competiçãa; melhoriade percepção em relação àssuas capacidades; ganho deautonomia; acesso alocaisque ofertem gratuitamente o esporte paralímpico (EP) parapessoas com comprometimento. De acordo com os ACC entrevistados, a permanência no EP gera sentido e significado às suas vidas. As barreiras mais citadas foram: desgaste físico; falta de val orização e reconhecimento como atletas; ausênciade patrocínio; dificuldades de acessibilidade; fal ta dedivulgação midiática. Este estudo oferece subsídios paraa construção de políticas públicas voltadas para o EP e para o trabalho de profissionais naárea.

Palavras-Chave:Atletascom comprometimentos; Facilitadores; Barreiras; Benefícios; Esporteparalímpico.
\end{abstract}

\begin{abstract}
The objective of the study was to explore the facilitators and barriers to the practice of sports by Brazilian elite athletes with impairments (AW I), actively involved in competitions at national and / or international level. The research was qual itative and exploratory. We collected the datathrough semi-structured interviews with nineAW I involved with six Paralympic sports. The main facilitators that they cited were: family support; example of other AW I through themedia; social ization; financial gains; incentivesfrom rehabilitation centersand health professionals; possibility of visiting new places; taste for sport and competition; improved perception of their capabilities; development of autonomy; access to placesthat offer freeParalympic sports (PS) for people with impairments. An important factor for the permanence of AW I in PS is that it generates meaning for their lives. The most cited barriers were: physical wear and injuries; lack of appreciation and recognition of athletes as sports professionals; lack of sponsorship; accessibility difficulties; lack of media coverage. Thisstudy offers subsidies for the development of public policiesfor PS and for the work of professionals in this field.
\end{abstract}

Key words: Athletes with impairments; Facilitators; Barriers; Benefits; Paralympic sports.

Resumen: El objetivo de este estudio fue explorar los facilitadoresY las barreras para la práctica de deportes por parte de atletas con discapacidad (ACD), brasileños, de alto rendimiento, activamente involucrados en competiciones anivel nacional y / o internacional. La investigación fue cualitativay exploratoria. Realizamos larecolección de datosatravés de entrevistas semiestructuradas anueveACD de seis deportes paralímpicos (DP). Los principal esfacilitadores mencionadosfueran: apoyo familiar; ejemplo de otrosACD através delos medios; socialización; beneficios económicos del deporte; incentivos de centros de rehabilitación y profesionales de la salud; posibilidad de visitar nuevos lugares; gusto por el deportey lacompetición; mejor percepción de suscapacidades desarrollo de laautonomía; acceso alugaresque ofrecen deportes gratuitosadaptados paraACD, yacceso al ugares libresy aptos paralos D P. U n factor importante paralapermanenciade los deportistas en el DP es que genera sentido y significado en sus vidas. Las barreras más citadas fueron: desgaste físico; falta de valoración y reconocimiento delosdeportistascomo profesionales del deporte; faltade patrocinio; dificultades deaccesibilidad; faltadedifusión mediática. Esteestudio ofrecesugerencias parala construcción de políticas públicasorientadasal D P y por el trabajo de los profesionalesen este campo. Palabrasclave: Atletascon discapacidad; Facilitadores; Barreras; Beneficios; D eporte paralímpico.

\section{Introdução}

No último senso demográfico brasileiro o Instituto Brasileiro de Geografiae Estatística(IBGE) (IBGE, 2010) identificou que $23,9 \%$ da população brasileira tem pelo

Fecha recepción: 22-10-20. Fecha de aceptación: 16-02-21

Yasmin Vicente Vieira

yasvvieira@outlook.com menos um tipo de comprometimento, seja ele visual, auditivo, motor, mental ou intelectual. Também de acordo com dados deste senso, reavaliados anos depois, 6,7\% da população possui comprometimento físico, auditivo, visual ou mental/ intelectual e têm «muita dificuldade» ou «não conseguem de modo algum» andar ou subir degraus, ouvir, enxergar, e/ ou realizar atividades habituais tais como brincar, ir à escola ou trabalhar (Simões et al., 2018). Do total da população, 37,9\% praticaal gum tipo de atividade física ou esportiva 
(IBGE, 2015). N ote que utilizamosa expressão «pessoas com comprometimento» (PCC) ao invés de «pessoas com deficiência». Embora no Brasil a grande maioria das pessoas continue utilizando a segunda expressão, optamos por seguir as recomendações do Comitê Paralímpico Internacional (IPC) (IPC, 2017) para a utilização do termo «impairment» (comprometimento) ao invés de «disability» (deficiência).

0 esporte paralímpico (EP) é uma das diferentes possibilidades de prática de exercício físico para PCC. Ele surgiu em um cenário pós-guerra, com o intuito de promover a reabilitação e melhoria da qual idade de vida dos soldados que adquiriram comprometimentos durante as batal has (Torri \& Vaz, 2017). Com o tempo, o EP cresceu em outras dimensões, inclusive no altorendimento, gerando oportunidades de profissional ização dos atletas (Benfica, 2012).

São vários os possíveis benefícios da prática esportiva para os atletas com comprometimentos (ACC), como por exemplo: oportunidades de socialização (Benfica, 2012; De Araújo, 2017; Fermino, 2020; Hutzler \& Bergman, 2011; Labronici et al., 2000; Martin, 2010; O liveiraetal., 2013; Pereiraet al. , 2013; Teodoro, 2006; Velasco et al., 2017; Wu \& W illiams, 2001); independência (Amorim et al., 2010; Benfica, 2012; Cardoso, 2011; Labronici et al., 2000; Pack et al., 2016; Pereira et al., 2013; Scherer et al., 2011; Velasco et al., 2017), qualidade de vida (Amorim et al., 2010; Benfica, 2012; Brazuna \& Mauerberg-deC astro, 2001; Cardoso, 2011; Pereira et al., 2013; Teodoro, 2006; Tsutsumi et al., 2004) melhoria das condições físicas e motoras (Scherer et al., 2011; Tsutsumi et al., 2004; Velasco et al., 2017), oportunidades econômicas, culturais e educacionais (Benfica, 2012; Florence, 2009); autoestima e/ ou autoconfiança e/ ou realização pessoal (Benfica, 2012; Cardoso, 2011; Costaet al., 2002; Fermino, 2020; Florence, 2009; Labronici et al., 2000; Martin, 2010; O liveira et al., 2013; Pack et al., 2016; Pereira et al., 2013; Ranieri, 2011; T sutsumi et al., 2004; Velasco et al. , 2017).

Ao entrar no esporte, muitos ACC se deparam com diversas condições que favorecem e/ ou limitam a participação e o desenvolvimento deles no meio esportivo. Neste contexto, 0 objetivo deste trabalho foi o de explorar os facilitadores e as barreiras para a prática esportiva por parte de ACC, brasileiros e de alto rendimento, ativamente envolvidos em competições de nível nacional ou internacional.

Alguns estudos vem discutindo os benefícios, significados, facilitadores e/ ou barreiras da prática esportiva
paraACC de alto rendimento (Benfica, 2012; Bragaru et al. ., 2013; Cardoso et al. , 2019; Coakley \& Pike, 2013; DaCosta et al., 2014; DeAraújo, 2017; Fermino, 2020; Hutzler \& Bergman, 2011; Jaarsma, 2014; Jaarsma, Dijkstra, et al., 2014; Jaarsma, Geertzen, et al., 2014; Samulski \& N oce, 2002; Scelza et al. , 2005; Stephens et al., 2012; Velasco et al., 2017; W ilson \& K hoo, 2013). No entanto, existe ainda a necessidade de mais estudos sobre estas temáticas, explorando-as a partir da perspectiva de atletas com diferentes graus de comprometimento, diferentes modalidades, e vivendo em diferentes contextos e local idades. Visamos com este estudo gerar conhecimentos que somem esforços neste sentido, com o intuito de subsidiar o trabalho de profissionais do esporte (gestores, treinadores, técnicos). Visamos também contribuir para com 0 desenvolvimento de políticas públicas que possam estimular e possibilitar o desenvolvimento esportivo no Brasil e em outras localidades com realidades similares.

\section{Métodos}

A pesquisa foi de cunho qual itativo e exploratório e aprovada pelo Comitê de Ética em Pesquisa da Universidade Federal do Paraná (CAAE: 68196517.1.0000.0102). A coleta de dados foi realizada através de entrevistas semiestruturas com nove ACC, de alto rendimento (três mulheres e seis homens), residentes na cidade de Curitiba/ PR, com idade entre 19 e 55 anos. Dentre eles, oito possuem comprometimento físico e um, comprometimento visual. Local izamos os atletas através de indicações de conhecidos e por meio dos próprios entrevistados que indicaram colegas. Incluímos no estudo apenas ACC envolvidos ativamente com o EP e que haviam participado de algum evento desportivo a nível nacional e/ ou internacional (ex. Jogos Paralímpicos Rio 2016, Jogos Parapan-Americanos de 2015 e J ogo Parapan-Americanos de 2019).

Após o esclarecimento dos procedimentos da pesquisa, os entrevistados assinaram um Termo de Consentimento Livree Esclarecido. A tabela 1 apresenta os participantes da pesquisa e al gumas das suas características. Ressaltamos que utilizamos os nomes reais dos entrevistados, uma vez que todos os participantes preferiram que não utilizássemos pseudônimos.

As entrevistas foram individuais e realizadas em sua maior parte presencialmente no local de treino dos ACC. Apenas uma entrevista foi realizada por Skype, devido à falta de disponibilidade do entrevistado para 

meus pais me dando 0 apoio financeiro eu não iria conseguir continuar» (TISBE, Natação).

Vários trabalhos apontam que a família é fundamental para 0 ingresso e permanência Fonte: Dados da pesquisa

realizá-la presencialmente. Elas duraram de 40 minutosaumahorae ocorreram entre os dias 27 de novembro de 2019 e nove de março de 2020. Realizamos as entrevistas a partir de um roteiro semiestruturado (tabela 2). Transcrevemos todas elas na íntegra e realizamos uma análise indutiva e temática dos dados (Braun \& Clarke, 2006), tendo como foco os principaistemas que emergiram da fala dos participantes.

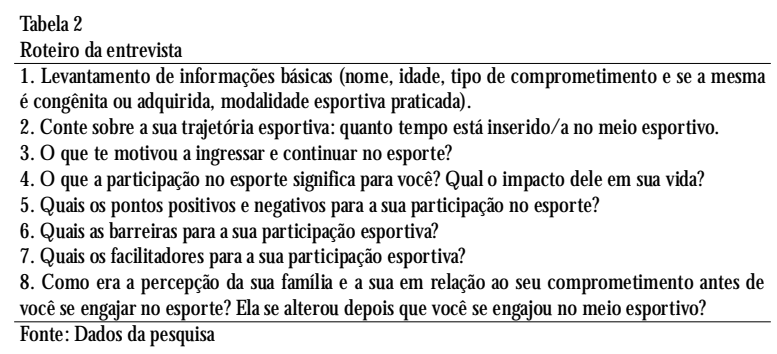

8. Como era a percepção da sua família e a sua em relação ao seu comprometimento antes de você se engajar no esporte? Ela se alterou depois que você se engajou no meio esportivo? Fonte: Dados da pesquisa

\section{Resultados e discussão}

O rganizamos 0 texto em duas partes. A primeira discute os facilitadores e a segunda as barreiras mencionados pelos ACC.

\section{Principais facilitadores para o envolvimento com OEP}

Para sete dos nove entrevistados, a família é um forte pilar de incentivo e apoio à prática esportiva.

«Eles [a família] me apoiaram totalmente!. . . . Vinham comigo para os treinos, para as competições. . .. A téhojeo meu irmão meajuda nos treinos» (ELISEU, Bocha).

«[A esposa] apoia muito. . . . Ela que me traz nos treinos, me orienta quando está certo e quando está errado. ... Q Q uando tem competições, ela está comigo viajando» (ALEXANDRE, Bocha).

Q uatro entrevistados declararam que o apoio financeiro oferecido pela família foi, e em al guns casos, continuasendo essencial paraqueeles pudessem ingressar e permanecer no EP.

«M inha mãe no começo foi minha patrocinadora máster. ... . Ela que arcou com tudo [financeiramente]» (JO ÃO LUCAS, Natação). no esporte (Benfica, 2012; Biduski et al., 2016; Carmona, 2015; Costa et al., 2002; De Araújo, 2017; Florence, 2009; Haiachi et al., 2016; Ranieri, 2011). O s incentivos (Brazuna \& M auerberg-deC astro, 2001; Buffart et al. , 2009; Cardoso et al., 2019; Carmona, 2015; Martin, 2010; Seron et al., 2015; Teodoro, 2006) e a ajuda financeira oferecida pelas famílias são essenciais até que 0 atleta consiga se destacar na modalidade e conquiste uma renda a partir de sua participação esportiva (Buffart et al., 2009; Teodoro, 2006). 0 apoio da família é também necessário para que os ACC consigam enfrentar as barreiras envolvidas na carreira esportiva (Campos et al., 2017; Cardoso et al., 2019; Haiachi et al., 2016; Jaarsma, Geertzen, et al., 2014; Teodoro, 2006). Conforme apontam al guns autores como Cardoso et al. (2019) eFermino (2020), aausênciado suporte dafamília pode trazer consequências negativas para a vida social e emocional do atleta, o que pode afetar a sua permanência no esporte e o desenvolvimento de suas habilidades esportivas. Mas ao mesmo tempo em que 0 apoio da família é importante, três entrevistados afirmaram que antes de entrarem no esporte, as suas famílias eram exageradamente protecionistas. Isto, deacordo com eles, pode ter atrasado um pouco o desenvolvimento da independência deles.

«Eles[afamília] acabaram sendo muito protecionistas. Até uns anos atrás . . . eu dependia muito deles [membros da família]. . . . Mas passou, hoje eu estou independente!. . . . Acho que eles tinham medo, por eu ter deficiência» (GABRIEL, Natação).

$O$ utro fator citado por sete entrevistados como motivador para a prática esportiva foi 0 exemplo de outros desportistas:

«M eu ídolo digamos assim. . . . ÉAlex M elo, campeão mundial da seleção brasileira. . . . Eu pensava: É nesse cara que eu vou me inspirar» (EVANDRO, Goalball).

Todos os entrevistados que adquiriram 0 comprometimento ao longo da vida mencionaram que a observação direta ou indireta (através da mídia) de outros atletas com realidades similares também os inspiraram para a prática. 
«0 Vinicius do atletismo, ele também teve a perna amputada! ... [Ele] sofreu um acidente parecido com o meu . . . . Amputou a perna . . . . E hoje ele é recordista mundial . . . . [lsso] me inspira muito, me dámotivação» (JOÃO LUCAS, Natação).

O s três ACC de natação que entrevistamos citaram Daniel Dias, atleta multimedal hista brasileiro como uma fonte motivadora para o ingresso e continuidade no EP. Vários estudos apontam que os exemplos e/ ou as conquistas de ACC podem se constituir como fontes de inspiração para PCC se engajarem e/ ou seguirem carreira no esporte (Cardoso et al., 2018; Coates \& Vickerman, 2016; Fermino, 2020; Haiachi et al., 2016; Silva \& Howe, 2012; Souza \& Brittain, 2021).

Para seis dos entrevistados, o contato com outros ACC através dos treinos e especialmente, em competições, é um importante meio de inspiração, incentivo e motivação para 0 engajamento e desenvolvimento deles no EP.

«N as competições a gente conhece pessoas novas, faz novas amizades. . . conhece pessoas de novos países» (GABRIEL, N atação).

0 envolvimento com o EP amplia o leque de convivência social das PCC (Fermino, 2020; K ristén et al. , 2002; Velasco et al., 2017). Isto, por sua vez, pode gerar um sentimento de pertencimento a outro grupo que não o familiar, o que pode melhorar a autoestima (Fermino, 2020; Haiachi et al., 2016; M auerbergdeCastro et al., 2016) o desenvolvimento de uma perspectiva mais positiva sobre as capacidades e possibilidades das mesmas (Swartz et al., 2018).

Devemos alertar, no entanto, que nem sempre a socialização é uma prioridade para os ACC de alto rendimento. Da Costa et al. (2014), por exemplo, ao estudarem um grupo de 25 atletas, concluíram que a busca por desempenho esportivo se sobressaia em relação à outros interesses tais como a social ização.

Seis entrevistados revelaram que têm o esporte como profissão, o que os motiva a permanecerem no EP.

«Eu tenho a carteira assinada com o cargo atleta. Consigo legitimar que realmente é um trabalho! . . . Me perguntam «qual a sua profissão? « Eu falo «atl eta»! Porque é uma profissão! . . . Q Querendo ou não são seis horas por dia, de segunda a sábado» (TISBE, N atação).

De acordo com o censo demográfico brasileiro (IBGE, 2010), a taxa de alfabetização dapopulação geral é de $90,6 \%$, enquanto das pessoas com pelo menos um comprometimento é de 81,7\%. Desta forma, elas têm mais dificuldade para se inserir no mercado de trabal ho.
Neste cenário, o esporte passa a ser uma opção profissional (Benfica, 2012). No entanto, esta opção não é desejadae nem acessível e/ ou possível a todos. M uitos não gostam da prática esportiva de alto rendimento e/ ou não têm acesso ao esporte (Torri \& Vaz, 2017). Vale também ressaltar que, se por um lado o esporte se constitui em uma via de inclusão social, por outro ele tende a segregar aqueles que não obtém sucesso (Cherney et al., 2015).

0 EP é a única fonte de renda para seis de nossos entrevistados:

«É meu trabal ho então eu tenho que estar aqui querendo ou não! . . . Às vezeseu não acordo com vontade de treinar, mas eu tenho que treinar, porque a minha evolução depende disso. . . . É minha fonte de renda» (JO ÃO LUCAS, Natação).

Do ponto de vista socioeconômico, muitas pessoas, principalmente das classes menos favorecidas, veem 0 esporte como um dos únicos meios de ascensão social (Viana \& M ezzaroba, 2013). No entanto, são muitas as barreiras para o desenvolvimento da carreira de um atleta (Haiachi et al., 2016; M auerberg-deC astro et al., 2016).

Seis entrevistados destacam que um dos principais motivos para o seu envolvimento com o esporte é 0 gosto pela prática esportiva e/ ou pela competição:

«0 que me motivou primeiramente é o gosto. . . . . Não consigo mais desgrudar [do esporte]» (EVANDRO, Goalball).

«Eu semprefui muito competitivo. ... Sempre gostei desse ambiente de competição, isso que me atraiu» (GABRIEL, Natação).

0 gosto pelo esporte pode levar a um envolvimento profundo com o mesmo, o que tende a ser fundamental paraque o atletapossa atingir bons resultados. Este gosto pode estar associado às oportunidades de superação que o EP oferece. Para algunsACC, 0 envolvimento com 0 esporte propicia com que eles compitam contra o seu comprometimento e contraos pensamentos construídos pela sociedade de que as PCC não são tão capazes quanto as pessoas sem comprometimentos (Brazuna \& Mauerberg-deC astro, 2001).

Cinco dos seis entrevistados que adquiriram 0 comprometimento ao longo da vida destacaram queeles conheceram ou se inseriram no meio esportivo graças ao acesso às informações e recomendações que receberam em associações de PCC e/ ou centros de reabilitação e/ ou através de profissionais da saúde.

«Eu estudava na associação dos deficientes visuais e eu ouvia muito sobre a modalidade de goal ball. . . . Em 
2009 eu fui atrás. Acabei gostando e estou até hoje» (EVANDRO, Goalball).

«Eu conheci o esporte no centro de reabilitação após asterapias, conhecendo al gumas modal idades de esporte, inclusive a que pratico hoje, a bocha» (ALEXANDRE, Bocha).

A disponibilização de informações sobre os benefícios da prática esportiva pode ajudar a motivar as PCC a conhecerem e a se engajarem neste meio (Buffart et al. , 2009; Sousa, 2014; Stancil, 2017; Swartz et al. , 2018; Teodoro, 2006; T sutsumi et al. , 2004; Wu \& W illiams, 2001). No entanto, nem sempre as associações de PCC e os profissionais da saúde têm conhecimento e/ ou repassam informações sobre a possibilidade da vivência esportiva por parte de PCC.

Seis entrevistados declararam que 0 envolvimento deles com o EP ajudou a mudar a percepção da família em relação aos seus potenciais e capacidades:

«Depois que eu entrei no esporte todo mundo conseguiu enxergar que eu sou uma pessoa capaz» (TISBE, N atação).

«Depois da minha entrada no esporte a visão deles [a família] ficou bem mais aberta. . . . Hoje eu sou independente» (GABRIEL, Natação).

A mudança de percepção das famílias tende a ocorrer quando os ACC obtém sucesso no esporte (Florence, 2009). A partir de então, a família tende a melhor apreciar as capacidades de seus parentes com comprometimento, que por sua vez, passam a ser um motivo de orgulho. Isto, consequentemente, contribui para com uma melhor aceitação do atleta em relação ao seu comprometimento (Pack et al., 2016; Sousa, 2014; Stephens et al., 2012; Teodoro, 2006). A vivência esportiva leva as PCC a desafiar e a interromper as restrições impostas pela família e por outras pessoas (Swartz et al., 2018). Ao superarem limitações, elas mostram que o comprometimento não é necessariamente al go trágico como a maioria das pessoas tende a pensar (Sousa, 2014; Swartz et al., 2018).

Q uatro entrevistados mencionaram que a percepção deles em relação às suas próprias capacidades se alterou para melhor depois que entraram no esporte. Tal como apontado pelos atletas no estudo de Swartz et al. (2018), eles deixaram de se ver como inferiores às pessoas sem comprometimentos:

«Eu consigo fazer absolutamente tudo [no esporte], tanto que uma pessoa sem deficiência se fosse jogar comigo, não daria tanta diferença assim» (EDIANDRO, Vôlei sentado).

A ampliação de horizontes através das viagens foi citada por cinco entrevistados como um fator que contribui para a permanência no esporte:

«Viajei bastante como nunca! . . . Com o esporte eu játive oportunidade de fazer viagensque eu nuncapensei em fazer» (EDIANDRO, Vôlei sentado).

«Conheci novos lugares. . . . As viagens ajudaram na minha evolução pessoal. . . . Ver novos lugares que eu nunca imaginei na vida ver» (GABRIEL, Natação).

0 esporte de alto rendimento pode proporcionar oportunidades de viagens (Benfica, 2012), o que para muitos, não seria possível se não fosse através do envolvimento com o esporte. Três entrevistados ressaltaram que o esporte os auxiliou no ganho de autonomia:

«Esporte para mim como deficiente representa a questão de autonomia, liberdade. . . . Porque você fez um esporte e tem uma noção espacial» (EVANDRO, Goalball).

0 atleta citado acima possui comprometimento visual. Ele revelou que a prática esportiva o ajudou a desenvolver uma melhor noção espacial, ajudando-0 a se deslocar com mais segurança e autonomia pela cidade. O utros estudos envolvendo PCC visual também apontaram este benefício da prática esportiva (Amorim et al., 2010; Pereira et al., 2013; Scherer et al., 2011; Velasco et al., 2017).

O utro fator motivador para a prática esportiva cita do por três entrevistados é o acesso a locais gratuitos e adaptados para a prática de esportes:

«0 projeto da (Pontifícia U niversidade Católica do Paraná) em si é um facilitador muito grande para as pessoas da comunidade de Curitiba e região. . . . N ormalmente tem que pagar e um projeto como esse dá a oportunidade sem ter que arcar com custos» (JO ÃO LUCAS, N atação).

Tendo em vista que muitas modalidades utilizam materiais com custos elevados, é importante que existam parcerias com locais apropriados e gratuitos parao desenvolvimento do esporte (A raújo et al. , 2020). Isto, por sua vez, contribui para que mais pessoas com e sem comprometimentos se engajem no esporte.

Por fim, mas não menos importante, ressaltamos que os nove entrevistados enfatizam que a participação no EP dá sentido e significado às suas vidas, 0 que os motiva a continuar no esporte.

«Q uando começou a aparecer a distrofia eu achava que tinha acabado a minha vida. . . . Depois que eu conheci o esporte ele completou minha vida de um jeito que eu não imaginava! . . . Ele [o esporte] devolveu sonhos que achei que não conseguiria mais» (ELISEU, 
Bocha).

«0 esporte éminhavida! . . É tudo o que eu tenho! . . . É meu objetivo pessoal! . . . Tenho que dar o meu máximo sempre» (GABRIEL, Natação).

«Todo atleta que realmente ama al gum esporte fala que ele significa tudo na vida. . . . Tudo que eu sou é graças a natação, eu construí a pessoa que eu sou [na natação]» (TISBE, N atação).

Três entrevistados revelaram ter pensado que a vida deles havia acabado após terem adquirido 0 comprometimento. Mas, com o apoio, inventivo da família, de outras pessoas e com a entrada no EP, perceberam que a vida continuaria, de outra forma.

\section{Principais barreiras para o envolvimento com 0 EP}

Ao falarem sobre as barreiras para 0 envolvimento no esporte, quatro participantes disseram que o EP de alto rendimento é o oposto de saúde, uma vez que desgasta 0 atleta e pode gerar lesões ao corpo:

«Ele [alto rendimento] mais prejudica o seu corpo do que traz bons aspectos para a saúde. . . . tem que buscar o seu máximo. . . . vocênão aguenta» (GABRIEL, Natação).

«Ele não é saúde, ele é o oposto! A gente vai muito ao limite físico, muscular e articular. . . . Isso gera riscos de lesões. . . . . No alto nível não adianta. Isso vai acontecer. . . . São consequências» (M ARINA, Paracanoagem).

M uitos acreditam que 0 esporte de alto rendimento gera benefícios paraasaúde (Viana\& M ezzaroba, 2013). Mas os treinosintensos, com cargas elevadas, bem como a superação de desafios com objetivo de obter recordes, são características importantes deste tipo de esporte. 0 esporte de al to rendimento exige o máximo do atleta do ponto de vista físico e psicológico (Benfica, 2012; Brazuna \& M auerberg-deC astro, 2001), podendo gerar lesões e malefícios para a saúde (Haiachi et al., 2016; Mauerberg-deC astro et al., 2016; Viana \& M ezzaroba, 2013). A busca incansável por medalhas pode levar 0 corpo ao limite, o que por sua vez, pode causar 0 abandono do esporte (Teodoro, 2006).

Q uatro entrevistados reclamaram da falta de visibilidade do EP na mídia. Isto, segundo eles, dificulta 0 acesso às informações a respeito do EP, e consequentemente, sobre as conquistas de ACC brasileiros.

«Está faltando mais visualização, mostrar mais o esporte para deficiente. ... . A gente só vê naTV o O límpico. . . . Tem muito deficiente que acha que não pode fazer nada, mas pode fazer muita coisa»
(EDIANDRO, Vôlei sentado).

«O esporte paralímpico no Brasil é muito mais qualificado [nas medalhas] . . . . Tem que ser mostrado! . . . N ão é possível que a mídia fique de olhos fechados para um detalhe desse» (EVANDRO, Goalball).

$N$ ão localizamos nenhum estudo que tenha investigado a visibilidade do EP na mídia brasileira, exceto durante a realização dos Jogos Paralímpicos (JP). Vale ressaltar que esta aumentou consideravelmente durante a edição Rio 2016, uma vez que o evento aconteceu no Brasil, o que gerou um maior interesse no mesmo (Santos et al., 2019; Souza \& Brittain, 2020). Após 0 evento, no entanto a visibilidade do EP diminuiu expressivamente. Mas al gumas mídias mantiveram para o EP um espaço um pouco maior do que tinham antes da candidatura do país para sediar os J ogos Paralímpicos Rio 2016 (Souza \& Brittain, 2020).

Q uatro entrevistados, tal como participantes de outros estudos, citaram a falta de patrocínio como uma importante barreira parao desenvolvimento deles como atletas. Eles também afirmaram que a falta de investimentos em atletas leva muitos a abandonarem 0 esporte.

«É muito difícil de conseguir patrocínio. . . . Então, quem não tem recurso não consegue ir pra frente» (TISBE, natação).

«A falta de patrocínio. ... M uitos atletas começam, as vezes tem futuro no esporte, só que por falta de investimentos. . . eles não conseguem chegar a um patamar alto no esporte. ... N ão ter patrocínio atrapal ha muito» (ELISEU, bocha).

«M uitos atletas não têm condições, por isso desistem» (CARMEN, Atletismo).

A escassez de patrocínio é uma grande barreira para 0 desenvolvimento de atletas (Benfica, 2012; Stephens et al., 2012), causando, em muitos casos, 0 abandono do esporte (Benfica, 2012; H aiachi et al. , 2016; M auerbergDeCastro et al. , 2016; Teodoro, 2006). A falta de incentivos financeiros para o EP está relacionada com diferentes fatores. Um deles é que o público tem pouco conhecimento sobre este tipo de esporte (MauerbergdeC astro et al., 2016). 0 u seja, não existe ainda uma culturaesportiva relacionadacom o EP que consigaatrair investimentos privados significativos para o EP. 0 utro fator, é a baixa visibilidade do EP na mídia. Existe uma estreita ligação entre esta baixa visibilidade e a capacidade dosórgãos gestores do EP delevantar fundos, conquistar patrocinadores e de melhorar as condições de marketing do EP (Brittain, 2010). Ainda outro fator que interfere com patrocínios é que muitos possíveis 
patrocinadores não desejam associar as suas marcas com 0 EP e nem com ACC, uma vez que os corpos destes atl etas se distanciarem do ideal de corpos bonitos, fortes eágeisque as marcas procuram (Faria\& C arvalho, 2011; Marques et al., 2014; Smith \& Bundon, 2018).

Cinco entrevistados são beneficiários do Programa Bolsa A tleta. Este programa, do Governo Federal Brasileiro, visa incentivar a carreira e 0 desempenho esportivo de atletas com bons resultados a nível nacional e internacional (Brasil, 2004). 0 número de bolsas disponíveis, no entanto, não atende à demanda. Além disto, os valores pagos são insuficientes para as despesas básicas de subsistência da grande maioria dos atletas. O s valores vão de R $\$ 370$ (o equivalente a aproximada mente 57 euros) a R\$ 15000 (2300 euros). A penas atletas com resultados expressivos a nível internacional e/ ou durante os JP chegam a receber bolsas com valores maiores que vão de $R \$ 1850$ (284 euros) a R \$ 15000 (2300 euros). Além destas bolsas que são fornecidas pelo Governo Federal, alguns atletas de alto desempenho podem também receber bolsas de Governos Estaduais e Municipais. No entanto, muitos desistem antes de chegar em níveis que lhes permitam pleitear este tipo de suporte. Dentre os que permanecem no esporte, quase sempre dependem do auxílio de suas famílias el ou de seu próprio trabal ho em outras áreas para que possam dar continuidade à sua carreira como atletas (M auerberg-deC astro et al., 2016).

Três entrevistados destacaram que outro fator que os desmotiva é a falta de reconhecimento, como profissional do esporte.

«As pessoas confundem com um hobby. .. você vem quando quer, nada quanto quer e vai embora. Alto rendimento não é bem assim! . . . Acham que por ser paralímpico é largado! . . . As pessoas desprezam o atleta paralímpico. . . . A Acham que é uma reabilitação. . . . . Não levam a sério! . . . Eu fico chateado porque desacreditam do meu trabalho» (JOÃO LUCAS, Natação, 43 anos).

A carênciade informações sobre o paradesporto gera uma visão distorcida a respeito do mesmo (M arques et al., 2013, 2014). Muitos 0 entendem como mera recreação. A falta de informações adequadas sobre os possíveis benefícios da prática esportiva por parte de PCC pode ser um dosfatoresqueinterferem no ingresso tardio deste público no esporte (Sousa, 2014).

Três participantes citaram problemas de acessibilidade e mobilidade urbana como barreiras para a prática esportiva:

«Tem vários lugares que não tem acesso. Ele [outro atl eta cadeirante] precisa de ajuda para subir. . . . ginásios por exemplo, nem todos têm acessibilidade. A maioria é feito só para quem consegue ter uma boa movimentação» (EDIANDRO, Vôlei sentado).

«Sofremos muito com isso! Asvezeso hotel échique, mas não tem banheiro adaptado ou só um liberado! . . . Muitas vezes os alojamentos não estavam preparados para nos receber» (CARM EN, Atletismo).

A falta de acessibilidade interfere diretamente na possibilidade de autonomia das PCC. Se as pessoas com comprometimento físico que utilizam cadeira de rodas encontrassem condições adequadas para se locomover, elas não precisariam da ajuda de outras pessoas para ir e vir livremente. A falta de acessibilidade impede que as PCC compareçam a determinados locais e eventos. Isto pode interferir negativamente em seu estado psicológico e contribuir para com a sua segregação e exclusão social (Benfica, 2012; Lima et al. , 2013; Silva \& Howe, 2012). A falta de acessibilidade e mobilidade urbana foram apontadas como barreiras parao desenvolvimento esportivo de PCC também em outras pesquisas (H aiachi et al., 2016; M auerberg-deC astro et al., 2016). Embora o Brasil tenha leis avançadas no sentido da promoção dos direitos das PCC, a efetiva implementação destas leis é ainda incipiente (Kirakosyan, 2016).

\section{Considerações finais}

Os principais facilitadores citados pelos entrevista dos paraingressarem e permanecerem no esporteforam: apoio motivacional e financeiro da família; inspirações geradas pelo exemplo de outrosACC através da mídia; social ização proporcionadaatravés do contato com novas pessoas; ganhos financeiros com o esporte; incentivos por parte de centros de reabilitação e / ou profissionais da saúde sobre os possíveis benefícios da prática esportiva; ampliação de horizontes através de viagens para competições; melhoria de auto percepção e da percepção de outras pessoas em relação às suas capacidades; aumento de autonomia gosto pelo esporte e/ ou pela competição; acesso a locais que ofertem gratuita mente EP. De acordo com todos os entrevistados, um grande fator motivacional para a sua permanência no esporte é que ele gera sentido e significado às suas vidas.

Asprincipaisbar reiras paraapermanência no esporte citadas pel os entrevistados foram: desgaste físico gerado pela prática esportiva de alto rendimento; baixa visibilidade do EP; falta de val orização e reconhecimento como atletas; fal ta de patrocínio; falta de acessibilidade. 
Entrevistamos nove ACC, de alto rendimento de seis modalidades em Curitiba, capital do Paraná, no Brasil. O s nossos resultados não podem ser general iza dos, uma vez que as realidades de atletas com diferentes tipos e graus de comprometimento, inseridos em diferentes modalidades e contextos socioculturais são diferentes. Mas eles nos permitem tecer algumas recomendações que podem ser úteis para a otimização de facilitadores e minimização de barreiras para 0 engajamento de PCC no EP de alto rendimento seja no Brasil ou em outros países com contextos similares.

Primeiramente, na mesma linha que outros autores como Haiachi et al. (2016) e Mauerberg deC astro et al. (2016), ressaltamos a necessidade da implementação de políticas públicas que fomentem o desenvolvimento do ACC desde a base. Estas políticas públicas precisam dar conta de questões básicas tais como a promoção de acessibilidade arquitetônica e de mobilidade urbana, criação e disponibilização de espaços e equipamentos adaptados e de livre acesso para PCC, e capacitação e contratação de profissionais qualificados para orientar a prática esportiva das PCC.

Faz-se também necessária uma maior divulgação do esporte para PCC e dos possíveis benefícios do engajamento no EP na mídia, escolas, centros de reabilitação e por via de profissionais da saúde. Desta forma, as PCC poderão se sentir mais motivadas - ou ser motivadas por seus pais ou responsáveis - a criarem hábitos saudáveis para a prática esportiva. U ma maior divulgação midiática do EP poderá também contribuir para com um maior reconhecimento das potencialida des e habilidades das PCC não só no esporte, mas também em outras atividades, ajudando na redução de estigmas e preconceitos relacionadas com as mesmas. Esta divul gação poderá também contribuir para que 0 EP vá pouco a pouco se tornando parte da cultura esportiva da população, o que poderá atrair mais praticantes e investimentos para o EP.

Para finalizar, gostaríamos de ressal tar a necessidade demaisestudosque explorem osfacilitadorese barreiras para a prática esportiva por parte de pessoas com diferentes tipos comprometimentos, de faixas etárias e contextos distintos, e engajadas em diferentes modalidades esportivas. Desta forma poderemos melhor entender as complexidades envolvidas para o efetivo engajamento das PCC no EP.

\section{Agradecimentos}

0 presente artigo foi realizado com apoio da bolsa de Iniciação Científica, PIBIC/ CNPq.

\section{Referências}

Amorim, M., Corredeira, R., Sampaio, E., Bastos, T., \& Botelho, M. (2010). Goalball: U ma modalidade desportivade competição. Revista Portuguesa de Ciências Do Desporto, 10 (1), 221-229. https:/ / doi.org/ 10.5628/ rpcd.10.01.221

Araújo, D. de S., Giordano, R. H., Lima, S. D. S., Gomes, J. F., Carvalho, I. P., Lima, E. B., Pereira, Silva, H. da, Guisande, J. D. R., Barros, N. P., \& Costa, L. G. T. (2020). Paracanoagem: Relato das ações de ensino, pesquisa e extensão desenvolvidas na U niversidade Federal do vale do São Francisco. Anais D o XI Congresso Brasileiro deAtividade M otora Adaptada (CBAMA), 7. https:/ / doity.com. br/ anais/ cbama2019/ trabalho/ 116625

Benfica, D. T. (2012). Esporte paraolímpico: analisando suas contribuições nas (re)significações do atleta com deficiência [(Dissertação de mestrado). U niversidade Federal de Viçosa, Minas Gerais, MG, Brasil]. https:/ / www. locus. ufv. br/ bitstream/ 123456789/3473/ 1/ texto completo.pdf

Biduski, G. M., Seron, B. B., Bertoli, J., Rossato, M., \& Freitas, C. de la R. (2016). Barreiras e facilitadores para a prática de esportes adaptados na U niversidade Federal de Santa Catarina. V Congresso Paradesportivo Internacional, 481-486. https:/ / doi.org/ 10.19177/ reen.v6e32013105-124

Bragaru, M., van W ilgen, C. P., Geertzen, J. H. B., Ruijs, S. G. J. B., Dijkstra, P. U., \& Dekker, R. (2013). Barriers and facilitators of participation in sports: A qualitative study on dutch individuals with lower limb amputation. Plos One, 8(3), 1-9. https:/ / doi.org/ 10.1371/ journal. pone. 0059881

Brasil. (2004). Ministério do Esporte. https:// www.gov. br/ cidadania/ pt-br/ acoes-e-programas/ bolsa-atleta

Braun, V., \& Clarke, V. (2006). Using thematic analysis in psychology. Qualitative Research in Psychology, 3(2), 77- 101. https:/ / doi. org/ https:/ / doi. org/ 10.1191/ 1478088706qp0630a

Brazuna, M. R., \& Mauerberg-deC astro, E. (2001). A trajetória do atleta portador de deficiência física no esporte adaptado de rendimento. U ma revisão da literatura. Motriz, 7(2), 115-123. http:// www.rc.unesp.br/ ib/ efisica/ motriz/ 07n2/ Brazuna.pdf

Brittain, I. (2010). Media, M arketing and Disability Sport. In The Paralympic Games Explained (pp. 72-90). Abingdon: Routledge.

Buffart, L. M., Westendorp, T., Van Den Berg-Emons, R. J., Stam, H. J., \& Roebroeck, M. E. (2009). 
Perceived barriers to and facilitators of physical activity in young adults with childhood-onset physical disabilities. Journal of Rehabilitation M edicine, 41(11), 881-885. https:/ / doi.org/ 10.2340/ 16501977-0420

Campos, R. C., Cappelle, M. C. A., \& Maciel, L. H. R. (2017). Carreira esportiva: 0 esporte de alto rendimento como trabalho, profissão e carreira. Revista Brasileira de Orientação Profissional, 18(1), 3141. http:/ / pepsic. bvsalud. org/ pdf/ rbop/ v18n1/ 04. pdf

Cardoso, V. D. (2011). A reabilitação de pessoas com deficiência através do desporto adaptado. Revista Brasileira de Ciências Do Esporte. Florianópolis, 33(2), 529-539. https:/ / doi.org/ 10.17058/ cinergis. v16i4. 6612

Cardoso, V. D., Haiachi, M. D. C., Poffo, B. N., Velasco, A. P., \& Gaya, A. C. A. (2018). A contribuição da mídia na construção dos ídolos paralímpicos brasileiros. Cadernos de Educação Tecnologia e Sociedade, 11(1), 78. https:// doi.org/ 10.14571/ brajets. v11.n1.78-86

Cardoso, V. D., Haiachi, M. D., Filho, A. R. R., \& Gaya, A. C. A. (2019). Motivos para a continuidade de atletas no esporte paralímpico brasileiro. Revista Iberoamericana de Psicologia D el Ejercicio y El D eporte, 14(1), 8-11. https:/ / www. riped-online. com/ articles/ reasons-for-continuity-of-athletes-in-brazilianparalympic-sport. pdf

Carmona, E. K. (2015). Atletas deficientes visuais sul-riograndenses nos jogos paralímpicos: Cenários e memórias [(Dissertação de mestrado). Universidade Federal do Rio Grande do Sul, Porto Alegre, RS, Brasil]. https:/ / lume. ufrgs. br/ bitstream/ handle/ 10183/ 127664/ 000974478. pdf?sequence=1\&isAllowed=y

Cherney, J. L., Lindemann, K., \& Hardin, M. (2015). Research in communication, disability, and sport. Communication and Sport, 3(1), 8-26. https:// doi.org/ 10.1177/ 2167479513514847

Coakley, J., \& Pike, E. (2013). Age and ability: Are they barriers to participation e inclusion in sports? In Age \& Ability in Sports. http:/ / www. pgedf.ufpr.br/ downloads/ Artigos PS M est 2018/ ELS/ Dora/ UFPR - Age $\&$ ability in sports.pdf

Coates, J., \& Vickerman, P. (2016). Paralympic legacy: exploring the impact of the Games on the perceptions of young people with disabilities people with disabilities. Adapted Physical Activity Quarterly, 33(4), 338- 357. https:/ / pubmed.ncbi.nlm. nih.gov/ 27874305/

ComitêParalímpico Internacional. (2017). Guia do Comitê Paralímpico Internacional (IPC). https:/ / ww w. paralympic. org/ sites/ default/ files/ d 0 c $\quad u \quad m$ e $n$ t l 170307082822939_2017_03_03+IPC+Style+Guide.pdf Costa, M. de O., Labronici, R. H. D. D., Mattos, E. de, Cunha, M. C. B., Oliveira, A. S. B., \& Gabbai, A. A. (2002). Bocha: Uma modalidade esportiva recreacional como método de reabilitação. Revista Neurociências, 10(1), 24-30. https:// periodicos. unifesp.br/ index.php/ neurociencias/ article/ view/8906/ 7582

Da Costa, L. C. A., Vissoci, J. R. N., M odesto, L. M., \& Vieira, L. F. (2014). 0 sentido do esporte para atletas de basquete em cadeiras de rodas: Processo de integração social e promoção de saúde. Rev. Bras. Ciênc. Esporte, Florianópolis, 36(1), 123-140. http:/ / www.scielo.br/ pdf/ rbce/ v36n1/ 0101-3289-rbce36-01-00123. pdf

De Araújo, A. C. S. (2017). Análise dos facilitadores e das barreiras para a prática de natação pela pessoa com deficiência física [(Dissertação de mestrado). Universidade de Brasília, Brasília, DF, Brasill]. https:/ / repositorio. unb. br/ bitstream/ 10482/ 24395/ 1/ 2017_AlessandraCristinaSilvadeA raújo.pdf

Faria, M. D. de, \& Carvalho, J. L. F. (2011). U ma análise semiótica do potencial mercadológico da imagem de atletas paraolímpicos. Gestão e Sociedade, 4(9), 32. https:/ / doi. org/ 10.21171/ ges. v4i9.994

Fermino, A. L. (2020). Os benefícios e os significados da participação em um grupo de bocha paralímpica no município de Curitiba/ PR [(Tese de doutorado). Universidade Federal do Paraná, Curitiba, PR, Bra sil]. $\quad$ https:/ / doi.org/ 10.1017/ CB0 9781107415324.004

Florence, R. B. P. (2009). Medalhistas de ouro nas paraolimpíadas deAtenas 2004: Reflexões de suas trajetórias no desporto adaptado [(Tese de doutorado). Universidade Estadual de Campinas, São Paulo, SP, Brasil]. http:/ / repositorio. unicamp. br/ jspui/ handle/ REPOSIP/ 274783

Haiachi, M. de C., Cardoso, V. D., Reppold Filho, A. R., \& Gaya, A. C. A. (2016). Reflections on the career of Brazilian paralympic athletes. Ciencia e Saude Coletiva, 21(10), 2999-3006. https:/ / doi.org/ 10.1590/ 1413-812320152110.18512016

Hutzler, Y., \& Bergman, U. (2011). Facilitators and barriers to participation while pursuing an athletic career: Retrospective accounts of swimmers with disabilities. Therapeutic Recreation Journal, 45(1), 116. https:/ / js. sagamorepub.com/ trj/ article/ view/ 29

Instituto Brasileiro de Geografia e Estatística (IBGE). (2010). Censo demográfico 2010: Características gerais da população, religião e pessoas com deficiência (IBGE (ed.)). 
https:/ / doi.org/ ISSN 0101-4234

Instituto Brasileiro de Geografia e Estatística (IBGE).

(2015). Pesquisa nacional por amostra de domicílios

(PN AD): Práticas de Esporte e atividade física. https:/ /

biblioteca. ibge. gov. br/ visualizacao/ livros/ liv100364.pdf

Jaarsma, E. A. (2014). Sports participation and physical disabilities: Taking the hurdle/ ?! https:// w w w. kennisbanksportenbew egen. nl/ ?file $=3688 \& m=1422883372 \&$ action=file. download Jaarsma, E. A., Dijkstra, P. U., Geertzen, J. H. B., \& Dekker, R. (2014). Barriers to and facilitators of sports participation for people with physical disabilities: A systematic review. Scandinavian Journal of Medicine and Science in Sports, 24(6), 871-881. https:/ / doi.org/ 10.1111/ sms. 12218

Jaarsma, E. A., Geertzen, J. H. B., de Jong, R., Dijkstra, P. U., \& D ekker, R. (2014). Barriers and facilitators of sports in Dutch Paralympic athletes: An explorative study. Scandinavian Journal of Medicine and Science in Sports, 24(5), 830-836. https:/ / doi.org/ 10.1111/ sms. 12071

Kirakosyan, L. (2016). Promoting disability rights for a stronger democracy in Brazil: The role of NGOS. N onprofit andVol untary Sector Quarterly, 45 (0489), 114S130S. https:/ / doi.org/ 10.1177/ 0899764015602129

Kristén, L., Patriksson, G., \& Fridlund, B. (2002). Conceptions of children and adolescents with physical disabilities about their participation in a sports programme. European Physical Education Review, 8(2), $139-156$. https:/ / doi. org/ 10.1177/ $1356336 \times 020082003$

Labronici, R. H. D. D., Cunha, M. C. B., De Oliveira, A. S. B., \& Gabbai, A. A. (2000). Esporte como fator de integração do deficiente físico na sociedade. Arquivos de N euro-Psiquiatria, 58(4), 1092-1099. https://doi.org/10.1590/s0004282x2000000600017

Lima, S. S. C., Carvalho-freitas, M. N., \& Santos, L. M. M. (2013). Repercussões psicossociais da acessibilidade urbana para as pessoas com deficiência física. Psico, Porto Alegre, 44(3), 362-371. https:// revistaseletronicas. pucrs. br/ ojs/ index. php/ revistapsico/ article/ view/ 15823/ 10411

Marques, R. F. R., Gutierrez, G. L., Almeida, M. A. B. de, \& Menezes, R. P. (2013). Mídia e o movimento paralímpico no Brasil: relações sob o ponto de vista de dirigentes do Comitê Paralímpico Brasileiro. Revista Brasileira deEducação Física eEsporte, 27(4), 583596. https:// doi.org/ 10.1590/ s180755092013000400007

Marques, R. F. R., Gutierrez, G. L., Almeida, M. A. B. de, Nunomura, M., \& M enezes, R. P. (2014). A abordagem midiática sobre o esporte paralímpico: perspectivas de atletas brasileiros. Movimento (ESEFID/ UFRGS), 20 (3), 989-1015. https:/ / doi.org/ 10.22456/ 1982-8918.41955

M artin, J. J. (2010). The psychosocial dynamics of youth disability sport. Sport Science Review, 19(5-6), 49-69. https: / / www. researchgate. net/ publication/ 265290884_The Psychosodid_Dynamics of YYouth__istality__Sport M auerberg-deC astro, E., Campbēll, D. F., \& Tavares, C. P. (2016). The global reality of the Paralympic M ovement: Challenges and opportunities in disability sports. Motriz. Revista de Educacao Fisica, 22(3), 111123. https:/ / doi.org/ 10.1590/ S19806574201600030001

Mauerberg-D eC astro, E., Figueiredo, G. A., lasi, T. C. P., \& Bagatini, L. (2016). Fatores que afetam a car reira esportiva de alto rendimento do atleta com deficiencia: U ma análise crítica. Revista Da Sobama, 17 (2), 23-30. https:/ / revistas. marilia. unesp. br/ index. php/ sobama/ article/ view/ 6827

Oliveira, C. H. S. de, Prada, A. C. B., Boato, E. M., Silva, J. V. P. da, Sampaio, T. M. V., \& Campbell, C. S. G. (2013). 0 goalball como possibilidade de inclusão social de pessoas Com deficiência visual. Pensar a Prática, 16(1), 165-182. https:/ / doi.org/ 10.5216/ rpp.v16i1.16460

Pack, S., Kelly, S., \& Arvinen-Barrow, M. (2016). «I think I became a swimmer rather than just someone with a disability swimming up and dow $\mathrm{n} /$ :» Paralympic athletes perceptions of self and identity development. Disability and Rehabilitation, 39(20), 2063-2070. https:// doi.org/ 10.1080/ 09638288.2016 .1217074

Pereira, R., O sborne, R., Pereira, A., \& Cabral, S. I. (2013). A importância do desporto de alto rendimento na inclusão social dos cegos: Um estudo centrado no Instituto Benjamin Constant - Brasil. Motricidade, 9(2), 94-105. https:/ / doi.org/ 10.6063/ motricidade. 9(2). 2671

Ranieri, L. P. (2011). Dimensões existenciais do esporte: Fenomenologia das experiências esportivas de atletas com deficiência visual [(Dissertação de mestrado). Univer sidade de São Paulo, São Paulo, SP, Brasil]. https:/ / www. teses. usp. br/ teses/ disponiveis/ 39/ 39134/tde-15042011-092854/publico/ dissertacao leandro ranieri. pdf

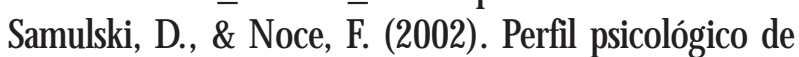 atletas paraolímpicos brasileiros. Revista Brasileira de M edicina D o Esporte, 8(4), 157-166. https:/ / doi.org/ 10.1590/ s1517-86922002000400005

Santos, S. M. dos, Furtado, S., Poffo, B. N., Velasco, A. 
P., \& Souza, D. L. de. (2019). Mídia e Jogos Paralímpicos no Brasil: a cobertura da Folha de $\mathrm{S}$. Paulo entre 1992 e 2016. Revista Brasileira de Ciencias Do Esporte, 41(2), 190-197. https:// doi.org/ 10.1016/ j.rbce.2018.03.012

Scelza, W. M., Kalpakjian, C. Z., Zemper, E. D., \& Tate, D. G. (2005). Perceived barriers to exercise in people with spinal cord injury. American Journal of Physical Medicine and Rehabilitation, 84(8), 576-583. h t t ps: / / doi.org/ 10.1097/ 01.phm. 0000171172.96290 .67

Scherer, R. L., Rodrigues, L. A., \& Fernandes, L. L. (2011). Contribuição do goalball para a orientação e mobilidade sob a percepção dos atletas de goalball. Pensar a Prática, 14(3), 1-15. https:/ / doi.org/ 10.5216/ rpp.v14i3.10777

Seron, B. B. , Arruda, G. A. De, \& Greguol, M. (2015). Facilitadores e barreiras percebidas para a prática de atividade física por pessoas com deficiência motora. Revista Brasileira de Ciencias Do Esporte, 37(3), 214- 221. j.rbce. 2013.09.003

Silva, C. F., \& Howe, P. D. (2012). The (In)validity of Supercrip Representation of Paralympian Athletes. Journal of Sport \& Social Issues, 36(2), 174- 194. https:/ / doi.org/ 10.1177/ 0193723511433865

Simões, A., Athias, L., \& Botelho, L. (2018). Panorama nacional e internacional da produção de indicadores sociais. Grupos populacionais específicos e uso do tempo. IBGE. https: / / biblioteca. ibge. gov. br/ visualizacao/ livros/ liv101562. pdf

Smith, B., \& Bundon, A. (2018). Disability models: Explaining and understanding disability sport in different ways. In I. Brittain \& A. Beacom (Eds.), The Palgrave Handbook of Paralympic Studies (1st ed., pp. 15-34). Palgrave Macmillan UK. https: / / doi. org/ 10.1057/ 978-1-137-47901-3

Sousa, A. I. C. A. e. (2014). A experiência vivida de atletas paralímpicos: Narrativas do desporto paralímpico português [(Tese de doutorado). Faculdade de Desporto da U niversiade do Porto, Porto, Portugal]. http:/ / cev.org. br/ arquivo/ biblioteca/ 4036941.pdf

Souza, D. L., \& Brittain, I. (2021). The Rio 2016 Paralympic Games: Inspiration as a possible legacy for disabled Brazilians legacy for disabled Brazilians. European Journal for Sport and Society, 0(0), 1-16. https:/ / doi. org/ 10.1080/ 16138171.2021.1879363

Souza, D. L. de, \& Brittain, I. (2020). The Rio 2016 Paralympic Games: The visibility of people with disabilities in Brazil as a possible legacy. Communication and Sport, 1-20. https:/ / doi.org/ $10.1177 / 2167479520942739$
Stancil, M. A. (2017). Physical activity and quality of life experienced by participants of a wheelchair basketball tournament [(D issertação de mestrado). U niversidade da Flórida, Gainesville,UF, Estados Unidos]. https:/ / pubmed.ncbi.nlm. nih. gov/ 18765861/

Stephens, C., Neil, R., \& Smith, P. (2012). The perceived benefits and barriers of sport in spinal cord injured individuals: A qualitative study. Disability and Rehabilitation, 34(24), 2061-2070. https:/ / doi.org/ $10.3109 / 09638288.2012 .669020$

Swartz, L., Bantjes, J., Knight, B., W ilmot, G., \& Derman, W. (2018). «They don't understand that we also exist»: South African participants in competitive disability sport and the politics of identity. Disability and Rehabilitation, 40(1), 35-41. https:/ / doi.org/ 10.1080/ 09638288.2016. 1242171

Teodoro, C. M. (2006). Esporte adaptado de alto rendimento praticado por pessoas com deficiência: Relatos de atletas paraolímpicos [(Dissertação de mestrado). Universidade Presbiteriana Mackenzie, São Paulo, SP, Brasil]. http:/ / tede. mackenzie. br/ jspui// handle/ tede/ 1600\# preview-link0

Torri, D., \& Vaz, A. F. (2017). Esporte paralímpico: Difícil inclusão, incorporação tecnológica, corpos competitivos. Práxis Educativa, 12(2), 1-15. https:/ / www. redalyc. org/ jatsR epo/ 894/ 89453001014/ $\mathrm{html} /$ index. html

Tsutsumi, 0., Cruz, S., Chiarello, B., Junior, D. B., \& Alouche, S. R. (2004). O sbenefícios danatação adaptada em indivíduos com lesões neurológicas. Revista N eurociências, 12(2), 82-86. http:/ / www. revistaneurociencias. com. br/ edicoes/2004/ RN 12 02/ Pages from RN 12 02-6. pdf

Velasco, A., Santos, S. M. dos, \& Souza, D. L. De. (2017). O s significados da prática do Goalball sob a ótica de atletas da modalidade. Alesde, 8(1), 43-58. https:/ / revistas. ufpr. br/ alesde/ article/ view/ 49977/ 35178

Viana, D. F. W., \& Mezzaroba, C. (2013). 0 esporte de alto rendimento faz mal à saúde? U ma análise das atletas da seleção brasileira de ginástica rítmica. M otrivivência, 0(41), 190-205. https:/ / doi. org/ 10.5007/ 2175-8042.2013v25n41p190

Wilson, N. C., \& Khoo, S. (2013). Benefits and barriers to sports participation for athletes with disabilities: The case of Malaysia. Disability and Society, 28(8), 1132-1145. https:// doi.org/ 10.1080/ 09687599.2012 .758034

Wu, S. K., \& W illiams, T. (2001). Factors influencing sport participation among athletes with spinal cord injury. M edicine and Science in Sports and Exercise, 33(2), 177-182. https:/ / pubmed. ncbi. nlm. nih. gov/ 11224802/ 